\title{
Design of Colloquial Arabic Electronic Atlas
}

\author{
Dong-Yul Lee ${ }^{1}$, Ji-Hoon Kang ${ }^{2}$, Sang-Ho Moon ${ }^{3}$ and Yong-Su Youn ${ }^{4}$ \\ ${ }^{1}$ Department of Global Area Studies, Graduate School Busan University of Foreign \\ Studies. \\ ${ }^{2}$ Institute of Mediterranean Studies. Busan University of Foreign Studies. \\ ${ }^{3}$ (Corresponding Author) Department of Computer Engineering, Busan University of \\ Foreign Studies. \\ ${ }^{4}$ Institute of Mediterranean Studies. Busan University of Foreign Studies. \\ ${ }^{1}$ akeram@bufs.ac.kr, ${ }^{2}$ mooswon@bufs.ac.kr, ${ }^{3}$ Shmoon87@bufs.ac.kr, \\ ${ }^{4} k a l i d @ b u f s . a c . k r$
}

\begin{abstract}
Arabic Language is characterized as a diglossia phenomenon, and that phenomenon base concept is Written Arabic and Colloquial Arabic. Colloquial Arabic is related with Arabic speaker's geographical location and living culture and history. In the paper we recommend research methodology using Electronic Atlas for Colloquial Arabic research. So we collect Colloquial Arabic Data and Database Design, and Design interface for Colloquial Arabic Electronic Atlas.
\end{abstract}

Keywords: Arabic language, Colloquial Arabic, Electronic Atlas, Diglossia, Research methodology

\section{Introduction}

Arabic language is one of the six official languages set by the United Nation, which is used by about 300 million people in 27 countries around world. Arabic language have special language character it is Diglossia phenomenon (bilingual phenomenon). Diglossia phenomenon defined by Ferguson, and that phenomenon is the upper variant of Arabic (Written Arabic, Modern Standard Arabic) and sub-variant of Arabic (Colloquial Arabic, Spoken Arabic) is coexistence in Arabic community and both are performing social functions. The concept has developed with the development of sociolinguistics, such as Triglossia, Multiglossia or Spectroglossia, which is based on Written Arabic and Colloquial Arabic.

Because of the Diglossa phenomenon of Arabic Language, Written Arabic's grammar and usage methods are same in Arabic community. But the colloquial Arabic show big difference by country or region. Basically, Arabic language are divided into Modern Standard Arabic (MSA, Written Arabic, اللغة العربية الفصحى) and colloquial Arabic (varieties of Arabic, Spoken Arabic, اللغة الغامية). In detail, Modern Standard Arabic means the modern Arabic language used in formal or formal situations based on classical Arabic, and colloquial Arabic is a spoken language used by Arabic community in informal situations. In the Arabic community, Modern Standard Arabic is commonly used as a standard language, and colloquial Arabic is used in carious forms depending on the country, region, society, class and gender.

Article history:

Received (January 21, 2018), Review Result (February 11, 2018), Accepted (March 23, 2018) 
The research about distribution and changes of colloquial Arabic can be an important tool for understanding the geographical location and living culture of the Arab region. In addition, it can be used in spoken language change research or research for inferring changes in language as a result of urbanization and various Arabic sociolinguistic studies. However, current research on the colloquial Arabic language is mainly dependent interviews and survey. These research methods have limitations in that they can only use limited data and it spend a lot of research time. In other words, individual researcher have disadvantage that it is difficult to obtain enough research data. And it is difficult to guarantee the representativeness of data.

To solve these problems, in the paper proposes a 'Colloquial Arabic Electronic Atlas' as a research method of Arabic sociolinguistics and colloquial Arabic language. To do this collect colloquial Arabic language textbook from major Arabic educational institutions or university. And also collecting and analyzing representative modern TV dramas of target Arab countries. We'll design the database by systematizing the regional distribution and variations for 'Colloquial Arabic Electronic Atlas'.

\section{Related research}

Research about classification colloquial Arabic has been performed in some foreign countries, but domestic studies are insufficient. As a representative overseas studies, Johnstone distinguished colloquial Arabic into Eastern colloquialisms and Western colloquialisms. In detail, Johnstone's classification distinguishes Maghreb (Algeria, Libya, Morocco, Tunisia and Mauritania) as Western colloquial classification and the rest of the region as Eastern colloquial classification. However, such a classification has limitation in that it cannot properly distinguish regional characteristics of colloquial Arabic language. And another research about classification colloquial Arabic is Versteegh's research. Versettgh categorized the colloquial Arabic as Arabian Peninsula, Mesopotamia, Syria-Lebanon, Egypt and Lebnon.

In domestic studies about colloquial Arabic language is focused on Egyptian colloquialism grammar. And there are few other studies are ongoing such as an education and colloquial Arabic general. Colloquial Arabic language research in Korea is follows.

Table 1. Colloquial Arabic Language studies in Korea

\begin{tabular}{|c|c|c|c|}
\hline Target Country & Frequency & Classification & Frequency \\
\hline Egypt & 20 & Grammar & 27 \\
\hline Jordan & 3 & Education & 2 \\
\hline Morocco & 2 & Literature & 7 \\
\hline Syria & 2 & General & - \\
\hline Bahrain & 1 & - & - \\
\hline Saudi Arabia & 1 & - & - \\
\hline Yemen & 1 & - & - \\
\hline Iraq & 1 & - & - \\
\hline Palestine & 1 & - & 42 \\
\hline General & 1 & - & Total \\
\hline Total & 42 & & - \\
\hline
\end{tabular}




\section{Collect colloquial Arabic language data}

Colloquial Arabic language studies in Korea are mainly based on interview and survey. This research method is useful for collection of colloquial Arabic language data use in each region, but there is limit to distribution of whole colloquial Arabic language and understand colloquial Arabic language use situation, and analyze discrimination each colloquial Arabic language. Subject of the paper are North Africa and East Mediterranean Area. Its six-country including Syria, Lebanon, Jordan, Egypt, Tunisia and Morocco. We collected colloquial Arabic language textbook from major Arabic educational institutions or university and Alexandria library in Egypt. So we chose 27 colloquial Arabic language textbook. In addition, we collect a popular TV drama set in the present from each country, which produced from each country. And collected colloquial Arabic from that TV dramas. This is because we think collect variation of major consonants in Arabic from textbook only, which is not proper. And we use TV dramas set in the present form each country, which produced from each country. Because even some TV dramas are popular in some country but produced other country can be possible and that TV drama did use that country's colloquial Arabic. And costume TV drama is usually played with Modern Standard Arabic so costume TV drama is not instance data to research colloquial Arabic but TV drama set in the present played with colloquial Arabic. Based on that 27 textbook and TV Drama set in the present that most popular and produced from each country, we design 'Colloquial Arabic Electronic Atlas'

That six countries have a colloquial Arabic language teaching institution form foreign students. And they are located in represent the characteristics of the Maghreb region (Major region of northern Africa, Tunisia, Morocco), Mashriq region (Eastern part of the Nile, Egypt) and Sham region (Levant, Syria, Jordan, Lebanon). These countries, historically have been colonized by different European counties and the characteristics of Colonial dominance in colloquial Arabic.

\section{Design of Database and Interface}

To construct colloquial Arabic Language information as a database, the international phonetic value of pronunciation of the colloquial Arabic phonetics are required for each Arabic consonant and vowel. To indicate the location in electronic atlas, location name, $\mathrm{x}$ and $\mathrm{y}$ coordinate values, which are position information of the point object, and $\left(\mathrm{x}_{1}, \mathrm{x}_{\mathrm{y}}\right) \ldots\left(\mathrm{x}_{\mathrm{n}}, \mathrm{y}_{\mathrm{n}}\right)$ coordinate values which are position information of the plane object are required. That information is indispensable information for display specific coordinate values on the atlas and superimpose the coordinate values of the specific Arabic consonant and vowel according to the same region. Basically, in order to distinguish the phonetic identity of each colloquium, international voice symbol value of the consonant of each Arabic consonant and vowel, country name for distinguish the location, DATA ID for use as the foreign key and the coordinate value of the point object and plane object. Use those information design metadata. Structuring a database for storing colloquial Arabic language information based on that metadata. The detailed schema is show in [Figure 1].

The interface of colloquial Arabic Language electronic atlas is shown in [Figure 2]. Pronunciation of the colloquial Arabic consonant and vowel on the atlas indicates the same area, and each area is displayed on the atlas using the point and plane coordinates. The pronunciation of each consonant and vowel is selected through drop box, and the selection result is designed to superimpose as [Figure 3].

Implementation of colloquial Arabic electronic atlas in this way, it can be useful for evaluating the similarity of the whole colloquial Arabic language because pronunciation of colloquial Arabic consonant and vowel can be selected by overlapping with regional display as [Figure 4]. This method will enable colloquial Arabic researcher to perform basic research tasks 
faster and easier than conventional methods. But researcher analyze and research the data obtained from electronic atlas is essential.

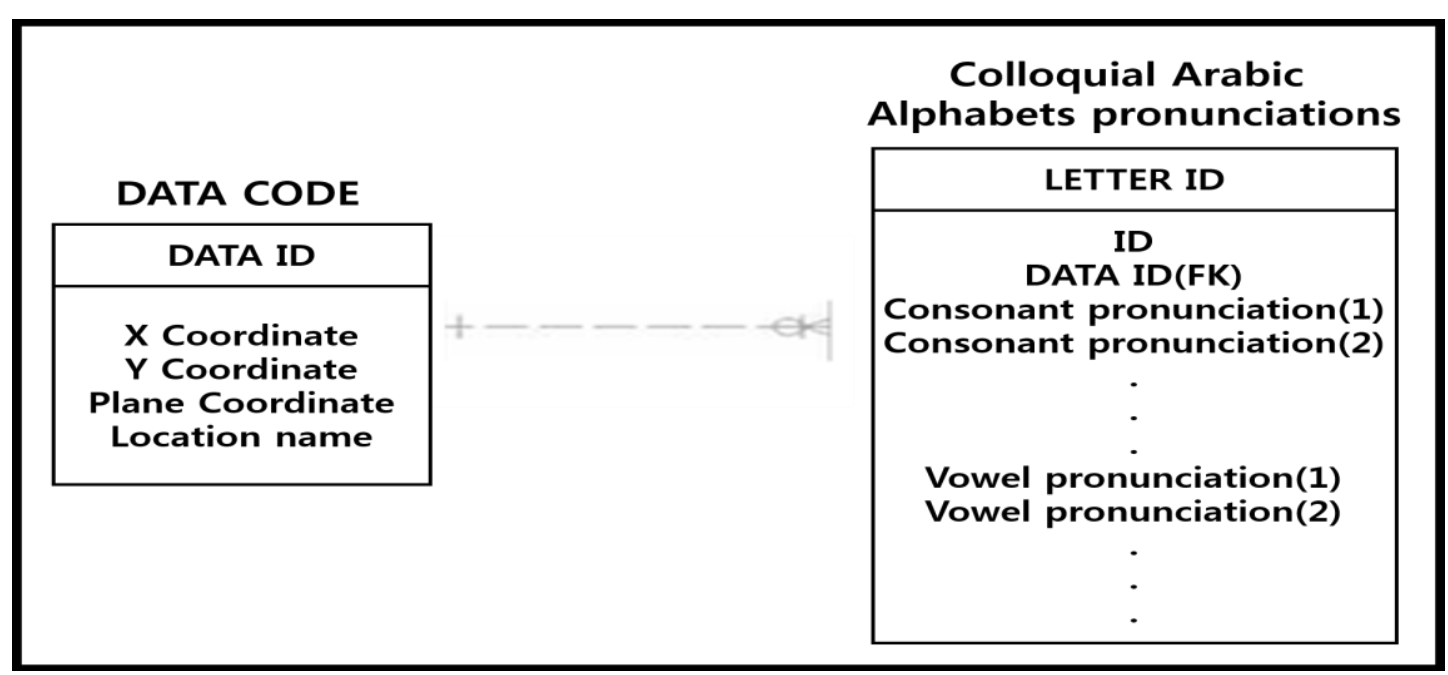

Figure 1. Database Schema for Colloquial Arabic Language

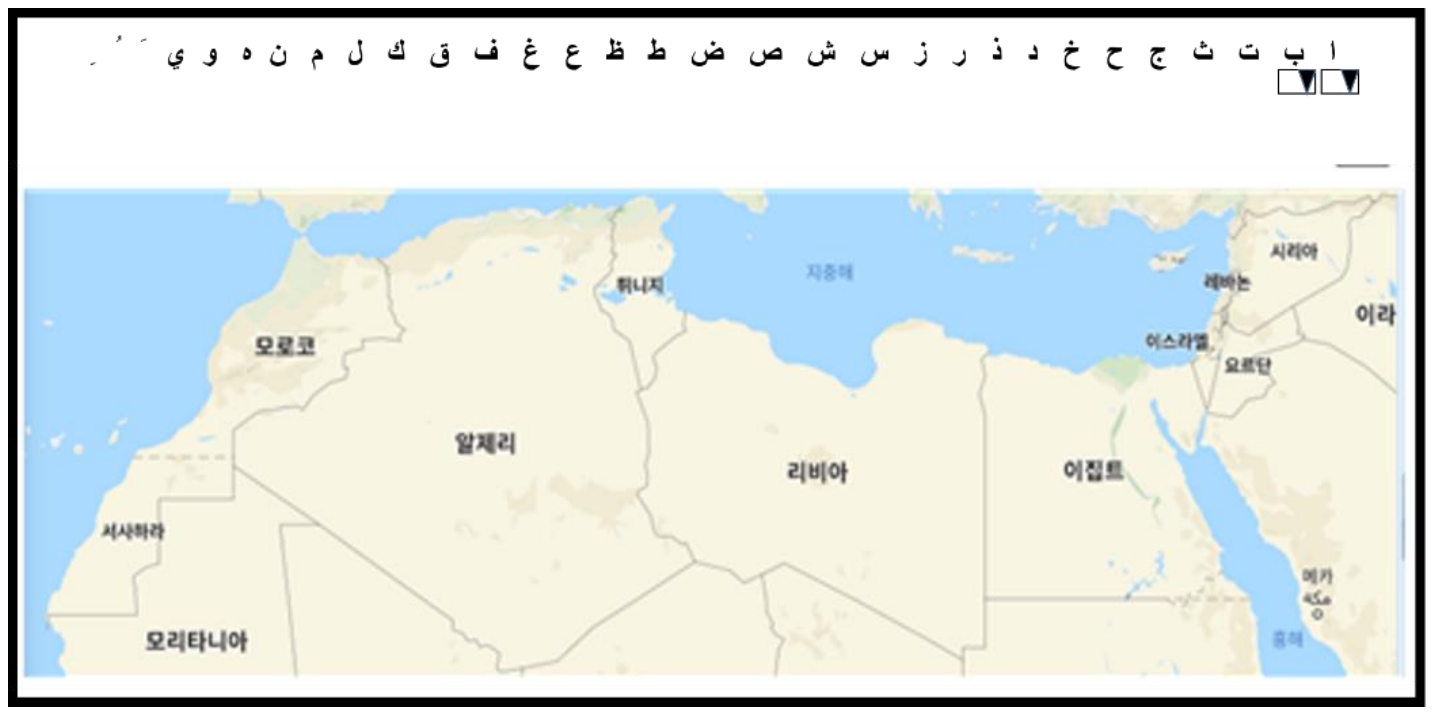

Figure 2. Page design of Electronic Atlas form Colloquial Arabic

\section{Conclusion}

In this paper, we design 'Colloquial Arabic Electronic Atlas' for proposing research methodology for colloquial Arabic research. We choose six country that have colloquial educational institutions and historically have been colonized by different European counties and the characteristics of Colonial dominance in colloquial Arabic such as Syria, Lebanon, Jordan, Egypt, Tunisia, and Morocco. From those countries collected and colloquial Arabic data such as educational text book, and TV dramas set in the present. From those materials extract pronunciation of the colloquial Arabic phonetics and match with international voice symbol value to construct colloquial Arabic database. Then use that database we design 
'Colloquial Arabic Electronic Atlas'. We guess this atlas can be useful for evaluating the similarity of the whole colloquial Arabic language. Future research will be carried out in the utility of the 'Colloquial Arabic Electronic' as research methodology.

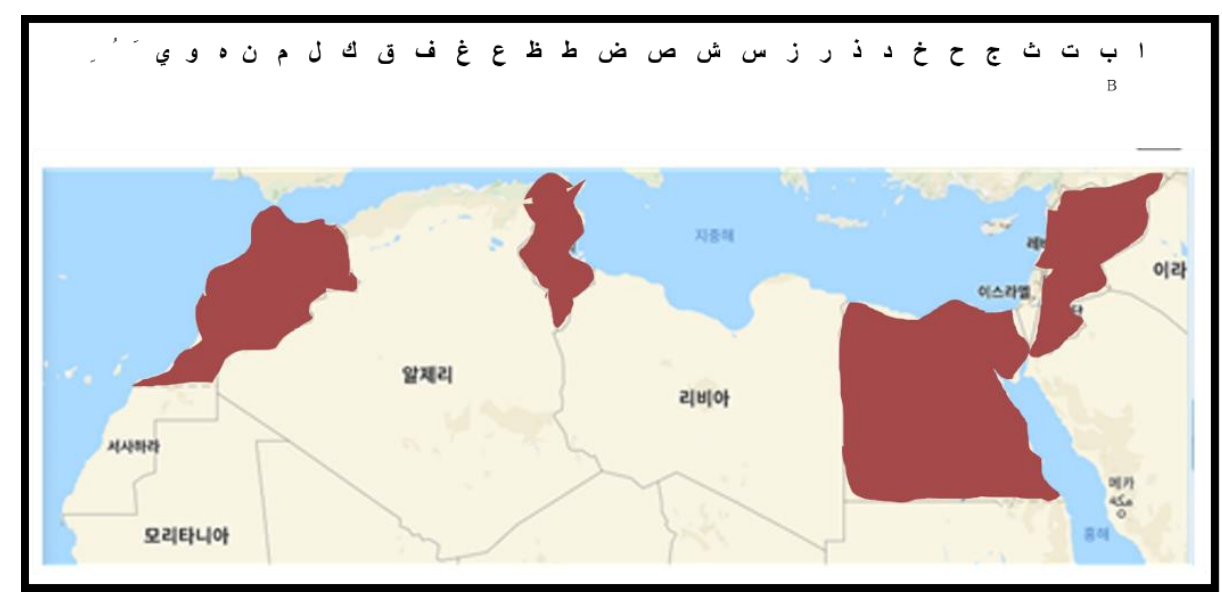

Figure 3. Page design of Electronic Atlas form Colloquial Arabic Choose as в

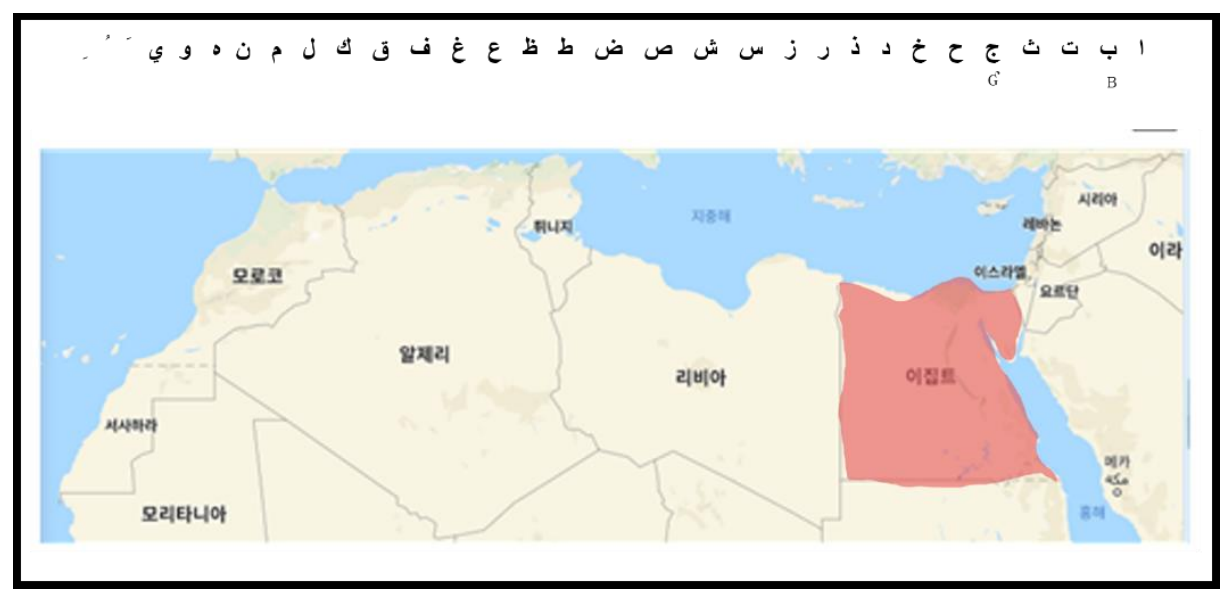

Figure 4. Page design of Electronic Atlas form Colloquial Arabic Choose as в and as $\mathrm{G}^{\mathrm{C}}$

\section{References}

[1] Ferguson Charles A., "Diglossia,” WORD, vol.15, no.1, (1959) DOI: 10.1080/00437956.1959.11659702

[2] Myung-Keun Oh, "A study on Verbal Tense and Conjugation in Spoken Arabic Focusing on Egyptian Spoken Arabic," Arabic language \& literature, vol.19, no.2, (2015)

[3] Eun-Kyeong Yun, "A Study on the concept and the features of Formal spoken Arabic," Arabic language \& literature, vol.7, no.1, (2003)

[4] Yong-Su Youn, "A Study on Cognizance of the Jordan Spoken Arabic," Arabic language \& literature, vol.7, no.2, (2003)

[5] Turki Ahmad Ali Bani-Khaled, "Standard Arabic and Diglossia: A Problem for Language Education in the Arab World," American International Journal of Contemporary Research, vol.4. no.8, (2014)

[6] Johnstone. T. M., Eastern Arabian dialect studies, Oxford University Press, London, (1967)

[7] Versteegh Kees, The Arabic language. Edinburgh University Press, Edinburgh, 2 edition, (2014) 
[8] Dong-Yul Lee, Ji-Hoon Kang, Chun-Sik Choi, Sang-Ho Moon, "A Study on Electronic Cultural Atlas using Thematic Overlay Function Focused on Israel-Palestine," Asia-pacific Journal of Multimedia Services Convergent with Art, Humanities, and Sociology, vol.5, no.3, (2015) 